\title{
Management of cardiovascular disease in patients with diabetes: the 2008 Canadian Diabetes Association guidelines
}

\author{
Onil K. Bhattacharyya MD PhD, Baiju R. Shah MD PhD, Gillian L. Booth MD MSc
}

Published at www.cmaj.ca on Sept. 18, 2008.

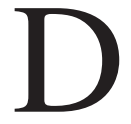
iabetes mellitus is a chronic condition that requires complex management; however, the time of health care providers is limited and patient motivation varies. How can health care providers design realistic treatment plans and establish priorities that maximize health benefits for patients? The 2008 Canadian Diabetes Association clinical practice guidelines ${ }^{1}$ (available online at www.diabetes.ca/for-professionals/resources/2008-cpg/) provide some suggestions to help meet these challenges. Although the guidelines cover a wide range of topics, our review is focused on the sections related to the management of cardiovascular disease.

Cardiovascular disease is the main cause of death among patients with diabetes mellitus. Over the last decade, there has been a 15\% decline in all-cause mortality and cardiovascular disease among people with diabetes, likely as a result of improved treatment and control of risk factors. ${ }^{2}$ Nevertheless, there is still room for improvement.

Guidelines on diabetes management have been developed by other national organizations, including the National Institute of Clinical Excellence 3 in the United Kingdom and the National Health and Medical Research Council in Australia. ${ }^{4}$ The Canadian Diabetes Association guidelines are updated every 5 years. They are subjected to an explicit review process focused on clinical evidence; however, these guidelines do not formally consider costeffectiveness or resource implications as do those developed by government bodies. ${ }^{3,4}$

Because risk factors for cardiovascular disease have a multiplicative effect, their reduction has a synergistic benefit for patients. ${ }^{5}$ The main interventions are controlling blood pressure, lipid levels and blood glucose, and promoting exercise, smoking cessation and healthy eating habits. ${ }^{1}$ These interventions are discussed below, except for smoking, which was not addressed in the 2008 guidelines, and diet, which cannot be easily summarized. ${ }^{6}$

In the following review, we consider the recommendations about the management of cardiovascular disease risk factors according to the magnitude of the health impact, strength of evidence, ease of implementation and how often they are fol-

\section{Key points}

- Cardiovascular disease is a key focus of the new diabetes guidelines.

- Men with diabetes aged 45 or more years and women with diabetes aged 50 or more years are at high risk of cardiovascular disease.

- The priorities for reducing risk of cardiovascular disease are control of blood pressure and cholesterol levels.

- Strict glycemic control can reduce microvascular complications, but it does not reduce the risk of cardiovascular disease.

- Control of cardiovascular disease risk factors is imperfectly implemented in current practice.

lowed, on average, compared with how often they could be followed. Average quality of care was obtained from a Health Quality Council report on diabetes in Saskatchewan from 2003 to $2004 .^{7}$ Because this province has universal insurance for pharmaceuticals and a centralized database for laboratory results, information about diabetes care is available for the entire population.

The best case scenario for clinical practice is based on the results of the Steno-2 trial. ${ }^{8,9}$ This randomized trial, performed at a diabetes centre with a team of health care providers (nurse, doctor, dietitian) and regular follow-up, reported that intensive control of risk factors reduced all-cause mortality by $46 \%$ compared with usual care. The staff and resources in this study may exceed those in many clinics, and the patient population included was at higher risk than people in the general population, however, Steno-2 is the only published large, long-term trial of a multifactorial intervention to prevent complications of diabetes. Thus, it serves as a "gold standard" for what may be achieved in clinical practice.

From the Keenan Research Centre, Li Ka Shing Knowledge Institute, St. Michael's Hospital (Bhattacharyya, Booth); the Department of Family and Community Medicine (Bhattacharyya) and the Department of Medicine (Shah, Booth), University of Toronto; the Institute for Clinical Evaluative Sciences (Shah, Booth); the Division of Endocrinology and Metabolism, Sunnybrook Health Sciences Centre (Shah), Toronto, Ont. 


\section{Methods used to develop the guidelines}

A comprehensive search of various electronic databases for relevant English-language, published, peer-reviewed studies was performed by chapter authors and independently by a librarian using validated search strategies. Full details of the search are included in the Canadian Diabetes Association guidelines. ${ }^{1}$ Each citation referenced in a new or modified recommendation was assigned a level of evidence by use of standardized checklists. Health benefits, risks and adverse outcomes of interventions were considered in the formulation of the recommendations. Recommendations were assigned a grade from A through D based on the relative strengths of the studies from a methodologic perspective and the studies' findings (Box 1, Table 1).

The guidelines underwent extensive external review by national and international experts in relevant fields and various stakeholder groups, including patients and health care professionals. A panel of methodologists independently reviewed each recommendation, its assigned grade and the supporting citations. Based on this review, each recommendation was reassessed and modified as necessary. Each recommendation was approved by the steering committee, with $100 \%$ consensus. Further details of the grading process have been described elsewhere. ${ }^{10}$

\section{Key elements of the $\mathbf{2 0 0 8}$ guidelines}

The 2008 updated guidelines build on the 2003 guidelines ${ }^{10}$ by updating the recommendations. These updates are based on newly available medications and newly published evidence, including large randomized controlled trials, such as Action in Diabetes and Vascular Disease: Preterax and Diamicron MR Controlled Evaluation (ADVANCE) ${ }^{11}$ and A Diabetes Outcome Progression Trial (ADOPT). ${ }^{12}$ New chapters have been added on hyperglycemic emergencies, in-hospital management of diabetes, alternative medicine, screening for coronary artery disease, diabetes mellitus and heart failure, and type 2 diabetes in children and high-risk ethnic populations. The recommendations reflect evidence of the average benefit of interventions in groups participating in clinical trials. These recommendations may not always apply to a particular patient, and care should be adapted to individual circumstances as necessary. ${ }^{13}$

\section{Cardiovascular risk}

The degree of benefit from a medical intervention is based on the absolute risk of vascular events, with patients at higher risk deriving greater benefit. ${ }^{7}$ The 2008 guidelines state that men aged 45 or more years with diabetes and women aged 50 or more years with diabetes should be considered to be at high risk of cardiovascular disease (grade B, level 2 evidence). This represents a $20 \%$ risk of vascular events over a 10 -year period, including nonfatal myocardial infarction or cardiac death. ${ }^{14}$ These age cutoff points are easy to ascertain and capture most patients at high risk who are most likely to benefit from intervention. Patients below these cutoff points who have 1 or more risk factors for cardiovascular disease are
Box 1: Levels of evidence for recommendations on the treatment and prevention of cardiovascular disease among people with diabetes

Level $1 \mathrm{~A}$

- Systematic overview or meta-analysis of high-quality randomized controlled trials

- Appropriately designed randomized controlled trial with adequate power to answer the question posed by the investigators

Level 1B

- Nonrandomized clinical trial or cohort study with indisputable results

Level 2

- Randomized controlled trial or systematic overview that does not meet level 1 criteria

Level 3

- Nonrandomized clinical trial or cohort study

Level 4

- Other

also considered to be at high risk (Box 2). This recommendation is more precise than that in the 2003 guideline, and it reflects a compromise between effectiveness (saving as many lives as possible) and efficiency (minimizing treatment given to people who will derive little or no benefit). Patients aged 40 or more years or with a more than 15-year history of diabetes should have baseline resting electrocardiography performed, and it should be repeated every 2 years for those at high risk (both grade $\mathrm{D}$, consensus). Patients with an abnormal electrocardiography result or chest pain should be referred for stress testing, and those with ischemia at low exercise capacity ( $<5$ metabolic equivalents) should be referred to a cardiologist (grade $\mathrm{D}$, consensus). The guidelines do not provide other explicit criteria for referral to a specialist.

\section{Vascular protection}

Certain medications have an independent effect on the risk of cardiovascular morbidity and mortality in addition to their effect on risk factors. The Heart Outcomes Prevention Evaluation (HOPE) study ${ }^{15}$ demonstrated the benefit of angiotensinconverting-enzyme (ACE) inhibition in people with diabetes who are at high risk for cardiovascular disease. A more recent study ${ }^{16}$ suggested that the effect of angiotensin-receptor blockers is comparable to that of ACE inhibitors in high-risk groups with diabetes. As a consequence, the updated guidelines recommend the use of ACE inhibitors or angiotensin-receptor blockers at

Table 1: Criteria for assigning grades to recommendations for clinical practice

\begin{tabular}{ll}
\hline Grade & \multicolumn{1}{c}{ Criterion } \\
\hline A & The best evidence is level 1 \\
B & The best evidence is level 2 \\
C & The best evidence is level 3 \\
D & The best evidence is level 4 or consensus \\
\hline
\end{tabular}


Box 2: Recommendations for assessing cardiovascular risk in people with diabetes'

Assessment of cardiovascular risk should be performed periodically and should include (grade $D$, consensus):

- History (dyspnea, chest discomfort)

- Lifestyle (smoking, level of activity, eating habits)

- Duration of diabetes

- Abdominal obesity

- Lipid profile

- Blood pressure

- Glycemic control

- Presence of retinopathy

- Estimated glomerular filtration rate and random albumin to creatinine ratio

The following people with diabetes should be considered at high risk for cardiovascular events:

- Men aged $\geq 45$ years, women aged $\geq 50$ years (grade B, level 2)

- Men $<45$ years and women $<50$ years with 1 of the following (grade $D$, consensus):

- Macrovascular disease

- Microvascular disease (especially nephropathy and retinopathy)

- Multiple additional risk factors:

- Family history of premature coronary or cerebrovascular disease in a first-degree relative

- Extreme level of a single risk factor (e.g., low-density lipoprotein cholesterol $>5.0 \mathrm{mmol} / \mathrm{L}$, systolic blood pressure > $180 \mathrm{~mm} \mathrm{Hg}$ )

- Duration of diabetes more than 15 years in a patient aged 30 years or older

doses shown to be cardioprotective for people at high risk of cardiovascular events, even in the absence of hypertension (grade A, level 1A for those with cardiovascular disease; grade $\mathrm{B}$, level 1A for those in other high-risk groups) (Box 3).

In Saskatchewan, 56\% of patients with diabetes are prescribed an ACE inhibitor or an angiotensin-receptor blocker. ${ }^{7}$ In comparison, $97 \%$ of patients in the Steno-2 study received an ACE inhibitor or an angiotensin-receptor blockers (even though the patients in this study all had microalbuminuria). ${ }^{8}$

The recommendation for acetylsalicylic acid (ASA) has been changed as a result of research showing it provides less benefit in terms of reduction of cardiovascular events among patients with diabetes than among those without diabetes. ${ }^{17,18}$ Thus, low-dose ASA therapy (81-325 mg/day) could be considered for people with stable cardiovascular disease (grade D, consensus). In primary prevention of cardiovascular disease, there is no evidence of a benefit of long-term ASA therapy among patients with diabetes; thus, its use is left to individual clinical judgment (grade $\mathrm{D}$, consensus).

\section{Blood pressure control}

Strict control of blood pressure has a large impact on morbidity and mortality in patients with diabetes. ${ }^{19-21}$ An observational study found that blood pressure control had a greater
Box 3: Recommendations for vascular protection in patients with diabetes ${ }^{1}$

- The first priority in the prevention of complications from diabetes should be reduction of overall cardiovascular risk by use of the following multifaceted approach (grade $D$ for all people with diabetes; grade A, level 1 for people aged 40 years or more with type 2 diabetes and microalbuminuria):

- For all people with diabetes:

- Lifestyle modification

- Achievement and maintenance of a healthy body weight

- Healthy diet

- Regular physical activity

- Smoking cessation

- Optimum blood pressure control

- Optimum glycemic control

- For people with diabetes who are considered at high risk of a cardiovascular event:

- ACE inhibitor therapy

- Lipid-lowering medication (primarily statins)

- People with diabetes who are at high risk for cardiovascular events should receive an ACE inhibitor (grade A, level 1 for people with diabetes, and coronary, cerebrovascular or peripheral arterial disease; grade $D$, consensus for other high-risk groups)

- Low-dose ASA therapy (81-325 mg/day) may be considered for people with stable cardiovascular disease (grade $\mathrm{D}$, consensus). Clopidogrel ( $75 \mathrm{mg} /$ day) may be considered for people unable to tolerate ASA (grade D, consensus). ASA for primary prevention of cardiovascular events should be based on individual clinical judgment (grade $D$, consensus)

Note: $\mathrm{ACE}=$ angiotensin-converting enzyme, ASA = acetylsalicylic acid.

impact on quality-adjusted life-years than strict control of cholesterol or glycemia. ${ }^{22}$ The 2008 guidelines recommend that blood pressure be measured at every patient visit (grade $\mathrm{D}$, consensus) and that hypertension be diagnosed according to the national hypertension guidelines (Box 4). ${ }^{23}$ The treatment targets remain a systolic blood pressure of less than $130 \mathrm{~mm} \mathrm{Hg}$ (grade C, level 3) and a diastolic pressure of less than $80 \mathrm{~mm} \mathrm{Hg}$ (grade B, level 2), irrespective of kidney function. First-line treatments include ACE inhibitors, angiotensin-receptor blockers, thiazides (grade A, level 1A for each) and dihydropyridine calcium-channel blockers (grade B, level 2). An ACE inhibitor or angiotensin-receptor blocker is recommended as initial treatment in the presence of microalbuminuria (grade A, level 1A). They are favoured because of demonstrated cardioprotective benefits (grade A, level 1A).

In clinical trials, achieving treatment targets has required more than 2 medications, ${ }^{24}$ and up to one-third of patients will require 3 or more medications with frequent titration. ${ }^{21,24} \mathrm{Al}-$ though no Canadian population-based data are available documenting hypertension control among patients with diabetes, a recent study ${ }^{25}$ reported that $66 \%$ of all patients with hypertension in Ontario had controlled blood pressure 
( $<140 / 90 \mathrm{~mm} \mathrm{Hg}$ ). Only $40 \%$ of Americans with diabetes meet the blood pressure target of less than 130/80 mm Hg. ${ }^{26}$ In the Steno- 2 trial, $51 \%$ of patients had a systolic pressure of less than $130 \mathrm{~mm} \mathrm{Hg}$ and $72 \%$ had a diastolic pressure less than $80 \mathrm{~mm} \mathrm{Hg.}{ }^{8}$ In that study, the difference in the proportion of patients at the target level between the intervention and control group was $30 \%$ for systolic blood pressure and 9\% for diastolic blood pressure, demonstrating considerable potential for improvement. ${ }^{8}$

Antihypertensive medications are well tolerated in general. Most hypertension trials have shown that treatment-related adverse outcomes are similar in frequency and severity among patients who received the drug and those who received a placebo. ${ }^{27}$ In fact, in the Hypertension Optimal Treatment (HOT) study, the arm aiming for a diastolic blood pressure of $80 \mathrm{~mm} \mathrm{Hg}$ had better patient well-being than the less-intensive arms, and well-being also increased with lower blood pressure across all treatment groups ${ }^{28}$ Although the updated guidelines do not specifically comment on the usefulness of home blood pressure monitoring, the Canadian Hypertension Education Program guidelines state that home monitoring should be considered for patients with diabetes. ${ }^{23}$ Furthermore, several studies have suggested that home blood pressure values are more predictive of mortality than blood pressure readings taken at office visits..$^{29,30}$

\section{Cholesterol management}

Cholesterol management by the use of statins reduces cardiovascular disease mortality by $19 \%$, regardless of baseline low-density lipoprotein (LDL) cholesterol levels. ${ }^{31,32}$ This was the finding for the subgroup of patients with diabetes with and without cardiovascular disease in a meta-analysis of large statin trials. ${ }^{31}$ The Canadian Diabetes Association cholesterol guidelines were released in $2006^{32}$ and remain unchanged. All patients at high risk of cardiovascular disease should take a statin to achieve an LDL cholesterol level of less than $2.0 \mathrm{mmol} / \mathrm{L}$ as a primary target (grade $\mathrm{A}$, level 1 ). A ratio of total cholesterol to high-density lipoprotein (HDL) cholesterol of less than 4.0 is a secondary target, which should be addressed with weight loss and increased physical activity (grade D, consensus). Ongoing clinical trials are evaluating a combination of statins and agents such as fibrates or niacin for patients whose ratio of total to HDL cholesterol is not at the target level. A recent study of the benefit of adding ezetimibe to statins for patients with familial hypercholesterolemia failed to show any benefit; thus, the role of combination therapy remains unclear. ${ }^{32,33}$

Statins are well tolerated and well-studied medications whose benefits largely outweigh the risks. ${ }^{31}$ Observational studies suggest similar effectiveness of various drugs in this class. ${ }^{34}$ The most common adverse outcome, seen in about $0.1 \%$ of patients who take statins for 1 year, is the reversible elevation of alanine or aspartate transaminase levels to more than 3 times the normal upper limit. ${ }^{35}$ Minor muscle pain was experienced equally by patients ( 1 in 20 ) in the placebo and control groups in randomized controlled trials. ${ }^{35}$ More severe adverse outcomes are rare: reversible myopathy (1 in 9100/year), rhabdomyolysis ( 1 in 29 000/year) and liver
Box 4: Recommendations for the treatment of hypertension in people with diabetes

- Blood pressure should be measured to assess hypertension at every visit to a diabetes clinic (grade D, consensus)

- Hypertension should be diagnosed according to national hypertension guidelines (grade D, consensus)

- People with diabetes and hypertension should receive treatment to attain a systolic blood pressure $<130 \mathrm{~mm} \mathrm{Hg}$ (grade C, level 3) and diastolic blood pressure $<80 \mathrm{~mm} \mathrm{Hg}$ (grade B, level 2). These target levels are the same as blood pressure treatment thresholds (grade D, consensus)

- Lifestyle interventions to reduce blood pressure should be considered, including achieving and maintaining a healthy weight, and limiting sodium and alcohol intake (grade D, consensus). Lifestyle changes should be initiated concurrently with pharmacologic interventions to reduce blood pressure (grade D, consensus)

- For people with normal kidney function, no microalbuminuria or macroalbuminuria, and blood pressure $>130 / 80 \mathrm{~mm} \mathrm{Hg}$ despite lifestyle interventions, any of the following medications are recommended* (particularly ACE inhibitors and angiotensin-receptor blockers):

- ACE inhibitor (grade A, level 1A)

- Angiotensin-receptor blocker (grade A, level 1A)

- Thiazide-like diuretic (grade A, level 1A)

- Dihydropyridine calcium channel blocker (grade B, level 2)

- For people with diabetes and albuminuria, an ACE inhibitor or an angiotensin-receptor blocker is recommended as initial therapy (grade A, level 1A)

- For people with diabetes, normal urinary albumin excretion, isolated systolic hypertension and no chronic kidney disease, a long-acting dihydropyridine calcium channel blocker (grade $C$, level 3 ) is an alternative initial choice rather than an ACE inhibitor (grade B, level 2), an angiotensin-receptor blocker (grade $B$, level 2) or a thiazide-like diuretic (grade $B$, level 2)

- Alpha-blockers are not recommended as first-line agents for the treatment of hypertension in people with diabetes (grade A, level $1 A$ )

Note: $\mathrm{ACE}=$ angiotensin-converting enzyme.

*If these drugs are contraindicated or cannot be tolerated, a

cardioselective beta-blocker (grade B, level 2) or a non-dihydropyridine calcium channel blocker (grade B, level 2) can be substituted. Another or more of these drugs should be added if target blood pressure levels are not achieved with standard-dose monotherapy (grade $\mathrm{C}$, level 3).

failure ( 1 in 200 000/year). ${ }^{35}$ However, the rate of liver failure in patients taking statins is similar to the rate in the general population. ${ }^{36}$

Despite the clear benefit of cholesterol control, only $45 \%$ of patients with diabetes in Saskatchewan had an LDL cholesterol level of less than $2.5 \mathrm{mmol} / \mathrm{L}$, and only $30 \%$ were taking lipid-lowering drugs. In the Steno-2 trial, ${ }^{9} 85 \%$ of patients in the intensive-treatment group were taking a statin, although this was a high-risk population. Given that choles- 
Box 5: Recommendations for physical activity in patients with diabetes

- People with diabetes should accumulate a minimum of 150 minutes of moderate to vigorous aerobic exercise each week, spread over at least 3 days, with no more than 2 consecutive days without exercise (grade B, level 2 for type 2 diabetes; grade C, level 3 for type 1 diabetes)

- People with diabetes (including elderly people) should be encouraged to perform resistance exercise 3 times a week (grade $B$, level 2 ) in addition to aerobic exercise (grade B, level 2). Initial instruction and periodic supervision by an exercise specialist are recommended (grade D, consensus)

- An exercise stress test should be considered for previously sedentary people with diabetes who are at high risk for cardiovascular disease and wish to undertake exercise more vigorous than brisk walking (grade D, consensus)

terol control often requires taking only 1 tablet each a day, with modest initial dose titration and few adverse outcomes, ${ }^{31}$ this medication is often an acceptable addition to the many drugs already taken by patients with diabetes.

\section{Glycemic control}

Elevated blood glucose is associated with microvascular and macrovascular complications. However, in randomized trials, improved glycemic control has resulted in reduced microvascular complications, such as nephropathy, but not in reduced cardiovascular events. ${ }^{11,20}$ The benefits of strict glycemic control are in question, as 1 arm of the Action to Control Cardiovascular Risk in Diabetes (ACCORD) trial, ${ }^{37,38}$ which was aimed at achieving a hemoglobin $\mathrm{A}_{1 \mathrm{c}}$ level of $6 \%$ or less, was stopped prematurely because of $22 \%$ higher mortality in the treatment group than in the less-intensive treatment group. Another recent study, the ADVANCE trial, ${ }^{11}$ showed that an intensive (modified-release) gliclazide-based regimen achieving a median hemoglobin $\mathrm{A}_{1 \mathrm{c}}$ level of $6.5 \%$ decreased nephropathy by $21 \%$ but did not decrease cardiovascular events compared with conventional treatment (which did not use gliclazide and achieved a median hemoglobin $\mathrm{A}_{\mathrm{cc}}$ of 7.3\%). The ADVANCE trial did not confirm an increased risk of death in the intensively controlled subgroup.

As a result, the updated guidelines recommend that glycemic targets be tailored to individual patients, but that a target hemoglobin $\mathrm{A}_{1 \mathrm{c}}$ level of $7.0 \%$ or less should be adopted for most patients with diabetes to reduce the risk of microvascular complications (grade $\mathrm{A}$, level 1A). The guidelines sugest that a target hemoglobin $\mathrm{A}_{1 \mathrm{c}}$ level of $6.5 \%$ or less may be considered for selected patients with type 2 diabetes to further lower the risk of nephropathy (grade A, level 1A), but the potential benefit must be balanced against the risk of hypoglycemia. Caution should be exercised for patients who are at significantly elevated risk of cardiovascular disease because of the increased mortality observed in the ACCORD study. ${ }^{38}$

Self-monitoring of blood glucose for patients with type 2 diabetes who do not take insulin was given a grade $\mathrm{C}$ recommendation because of the contradictory evidence about the benefit, including a recent randomized controlled trial and older meta-analysis that showed no benefit. ${ }^{39,40}$ A recent trial reported that in addition to having no benefit in patients not taking insulin, self-monitoring caused a small increase in patient scores on the depression subscale of a questionnaire used to gauge well-being. ${ }^{41}$ For example, glucose test strips are the fourth most expensive "chemical" in the Ontario Drug Benefit program; ${ }^{42}$ thus, the opportunity cost of this intervention should be considered.

Achieving glycemic control based on a target of hemoglobin A1c level of $7 \%$ or lower may require multiple medications and has been achieved only inconsistently. In Saskatchewan, only $38 \%$ of patients had a hemoglobin $\mathrm{A}_{1 \mathrm{c}}$ lower than $7 \% .^{7}$ In the Steno- 2 trial, ${ }^{8}$ only $16 \%$ of patients in the intervention group achieved the target of a hemoglobin $\mathrm{A}_{1 \mathrm{c}}$ level of less than $6.5 \%$, suggesting that, even under ideal conditions, strict glycemic control is difficult to achieve. Despite the fact that this target was not achieved for $84 \%$ of patients, the intensive-therapy group in the Steno-2 study still displayed dramatic reductions in cardiovascular and all-cause mortality. ${ }^{9}$

\section{Exercise promotion}

Large cohort studies have found that regular physical activity is associated with 39\%-70\% reductions in cardiovascular and all-cause mortality over 15-20 years of follow-up. ${ }^{43-46}$ The effect of simply recommending that patients exercise more (which is what most clinicians do) is less certain. ${ }^{47}$ Structured physical activity counselling by physicians or health care providers has been effective in increasing physical activity and producing modest, sustained weight loss. ${ }^{48-51}$ The recommendation of 150 minutes of moderate to vigorous aerobic exercise spread over 3 days a week (grade B, level 2) is unchanged from the 2003 guidelines (Box 5). The guidelines also recommend resistance exercise 3 times a week in addition to aerobic exercise (grade B, level 2); resistance exercise can be helpful for people with limited mobility. Finally, a cardiac stress test should be considered for previously sedentary people at high risk of cardiovascular disease who wish to undertake exercise more vigorous than brisk walking (grade $\mathrm{D}$, consensus).

\section{What is the best way to reduce the risk of cardiovascular disease?}

Given the limited time available for regular primary care visits and the range of risk-reduction strategies, it is best to explain the options to patients and to build a long-term treatment plan.

Blood pressure and cholesterol control are easiest to implement and have a seemingly greater health impact; thus, these should have priority over strict glycemic control. Using multiple medications at lower doses can optimize risk-factor control while minimizing adverse outcomes. ${ }^{52}$ Patients who have elevated blood pressure or who do not want to take "too 
many pills" can take combination therapies, which may help them attain targets quickly. ${ }^{53}$

Self-monitoring of blood glucose for patients not taking insulin is of uncertain benefit; thus, other behaviours, such as exercise, should be promoted. Consider offering outside resources to support behaviour change, such as exercise programs for motivated patients.

Overall, quality of care can be improved through the use of electronic databases, clinical flow charts and automatic reminders (grade B, level 2). Enhancing the role of nurse educators, dietitians and pharmacists can improve coordination of care (grade B, level 2). Case management should be considered for difficult-to-manage cases (grade B, level 2).

In conclusion, because cardiovascular disease is the main cause of mortality among diabetic patients, managing cardiovascular risk should be a key treatment goal for providers and patients.

This article has been peer reviewed.

Competing interests: None declared.

Contributors: Onil Bhattacharyya wrote the first draft of the article. He, along with Baiju Shah and Gillian Booth, revised the manuscript critically for intellectual content and approved the final version submitted for publication.

Acknowledgements: Baiju Shah has received funding from the Canadian Institutes of Health Research, the Canadian Diabetes Association and the Banting and Best Diabetes Centre at the University of Toronto. Gillian Booth has received funding from the Canadian Institutes of Health Research, the Ontario Women's Health Council and the Banting and Best Diabetes Centre at the University of Toronto.

Funding: This authors not receive external funding for this article. It was written independently of the Canadian Diabetes Association guidelines.

Editor's note: Gillian Booth is a member of the steering and executive committees for the 2008 Canadian Diabetes Association Clinical Practice Guidelines and was editor of the Methods section of the guidelines. Onil Bhattacharyya and Baiju Shah were not involved in the writing of the 2008 Canadian Diabetes Association Clinical Practice Guidelines but are members of the dissemination and implementation subcommittee.

\section{REFERENCES}

1. Canadian Diabetes Association Clinical Practice Guidelines Expert Committee. Canadian Diabetes Association 2008 clinical practice guidelines for the prevention and management of diabetes in Canada. Can J Diabetes 2008;32(suppl 1):S1-201. Available: www.diabetes.ca/for-professionals/resources/2008-cpg/

2. Booth GL, Kapral MK, Fung K, et al. Recent trends in cardiovascular complications among men and women with and without diabetes. Diabetes Care 2006;29:32-7.

3. National Institute for Clinical Excellence. The guideline development process: an overview for stakeholders, public and the NHS (3rd ed). London (UK): The Institute; 2007. Available: www.nice.org.uk/media/830/B9/GuidelinesManualDevelopmentProcess.pdf (accessed 2008 Sep 6).

4. Australian Centre for Diabetes Strategies. Introduction and overview of the guideline development process. In: National evidence based guidelines for the management of type 2 diabetes mellitus. Sydney (Australia): National Health and Medical Research Council; 2005. Available: www.nhmrc.gov.au/publications/synopses /_files/di7.pdf (accessed 2008 Sep 6).

5. Jackson R, Lawes CM, Bennett DA, et al. Treatment with drugs to lower blood pressure and blood cholesterol based on an individual's absolute cardiovascular risk. Lancet 2005;365:434-41.

6. Van Horn L, McCoin M, Kris-Etherton PM, et al. The evidence for dietary prevention and treatment of cardiovascular disease. J Am Diet Assoc 2008;108:287-331.

7. Chan BT, Klomp H, Castagnette P. Quality of diabetes management in Saskatchewan. Saskatoon (SK): Health Quality Council; 2006.

8. Gaede P, Vedel P, Larsen N, et al. Multifactorial intervention and cardiovascular disease in patients with type 2 diabetes. N Engl J Med 2003;348:383-93.

9. Gaede P, Lund-Andersen H, Parving HH, et al. Effect of a multifactorial intervention on mortality in type 2 diabetes. N Engl J Med 2008;358:580-91.

10. Canadian Diabetes Association Clinical Practice Guidelines Expert Committee.
Canadian Diabetes Association 2003 clinical practice guidelines for the prevention and management of diabetes in Canada. Can J Diabetes 2003; 27:(suppl 2). Available: www.diabetes.ca/cpg2003/downloads/cpgcomplete.pdf (accessed 2008 Sep 6).

11. The ADVANCE Collaborative Group. Intensive blood glucose control and vascular outcomes in patients with type 2 diabetes. N Engl J Med 2008;358:2560-72.

12. Kahn SE, Haffner SM, Heise MA, et al. Glycemic durability of rosiglitazone, metformin or glyburide monotherapy. N Engl J Med 2006;355:2427-43.

13. Woolf SH, Grol R, Hutchinson A, et al. Clinical guidelines: potential benefits, limitations, and harms of clinical guidelines. BMJ 1999;318:527-30.

14. Booth GL, Kapral MK, Fung K, et al. Relation between age and cardiovascular disease in men and women with diabetes compared with non-diabetic people: a population-based retrospective cohort study. Lancet 2006;368:29-36.

15. Yusuf S, Sleight $P$, Pogue J, et al. Effects of an angiotensin-converting-enzyme inhibitor, ramipril, on cardiovascular events in high-risk patients. Heart Outcomes Prevention Evaluation Study Investigators. N Engl J Med 2000;342:145-53.

16. ONTARGET Investigators, Yusuf $\mathrm{S}$, Teo KK, et al. Telmisartan, ramipril, or both in patients at high risk for vascular events. N Engl J Med 2008;358:1547-59.

17. Aspirin effects on mortality and morbidity in patients with diabetes mellitus. Early Treatment Diabetic Retinopathy Study report 14. ETDRS Investigators. JAMA 1992;268:1292-300.

18. Collaborative meta-analysis of randomised trials of antiplatelet therapy for prevention of death, myocardial infarction, and stroke in high risk patients. BMJ 2002; 324:71-86.

19. The CDC Diabetes Cost-effectiveness Group. Cost-effectiveness of intensive glycemic control, intensified hypertension control and serum cholesterol level reduction for type 2 diabetes. JAMA 2002;287:2542-51.

20. UK Prospective Diabetes Study Group. Intensive blood-glucose control with sulphonylureas or insulin compared with conventional treatment and risk of complications in patients with type 2 diabetes: UKPDS 33. Lancet 1998;352:837-53.

21. UK Prospective Diabetes Study Group. Tight blood pressure control and risk of macrovascular and microvascular complications in type 2 diabetes: UKPDS 38 . BMJ 1998;317:703-13.

22. Schmittdiel J, Vijan S, Fireman B, et al. Predicted quality-adjusted life years as a composite measure of the clinical value of diabetes risk factor control. Med Care 2007:45:315-21.

23. 2008 CHEP recommendations for the management of hypertension. Canadian Hypertension Education Program (CHEP); 2008. Available: hypertension.ca/chep /wp-content/uploads/2008/03/2008-chepspiral-booklet-final_jan28.pdf (accessed 2008 Sep 6).

24. Whelton PK, Barzilay J, Cushman WC, et al. Clinical outcomes in antihypertensive treatment of type 2 diabetes, impaired fasting glucose concentration, and normoglycemia: Antihypertensive and Lipid-Lowering Treatment to Prevent Heart Attack Trial (ALLHAT). Arch Intern Med 2005;165:1401-9.

25. Leenen FH, Dumais J, McInnis NH, et al. Results of the Ontario survey on the prevalence and control of hypertension. CMAJ 2008;178:1441-9.

26. Resnick HE, Foster GL, Bardsley J, et al. Achievement of American Diabetes Association clinical practice recommendations among U.S. adults with diabetes, 1999-2002 the National Health and Nutrition Examination Survey. Diabetes Care 2006;29:531-7.

27. Bremner AD. Antihypertensive medication and quality of life - Silent treatment of a silent killer? Cardiovasc Drugs Ther 2002;16:353-64.

28. Hansson L, Zanchetti A, Carruthers SG, et al. Effects of intensive blood-pressure lowering and low-dose aspirin in patients with hypertension: principal results of the Hypertension Optimal Treatment (HOT) randomised trial. HOT Study Group. Lancet 1998;351:1755-62.

29. Bobrie G, Chatellier G, Genes N, et al. Cardiovascular prognosis of "masked hypertension" detected by blood pressure self-measurement in elderly treated hypertensive patients. JAMA 2004;291:1342-9.

30. Verberk WJ, Kroon AA, Kessels AG, et al. Home blood pressure measurement: a systematic review. J Am Coll Cardiol 2005;46:743-51.

31. Baigent C, Keech A, Kearney PM, et al. Efficacy and safety of cholesterol-lowering treatment: prospective meta-analysis of data from 90,056 participants in 14 randomised trials of statins. Lancet 2005;366:1267-78.

32. Canadian Diabetes Association Clinical Practice Guidelines Expert Committee Dyslipidemia in adults with diabetes. Can J Diabetes 2006;30:230-40. Available: www.diabetes.ca/files/CJDSep06Lipids.pdf (accessed 2008 Sep 6).

33. Kastelein JJP, Akdim F, Stroes ESG, et al. Simvastatin with or without ezetimibe in familial hypercholesterolemia. $N$ Engl J Med 2008;358:1431-43.

34. Zhou Z, Rahme E, Abrahamowicz M, et al. Effectiveness of statins for secondary prevention in elderly patients after acute myocardial infarction: an evaluation of class effect. CMAJ 2005;172:1187-94.

35. Law M, Rudnicka AR. Statin safety: a systematic review. Am J Cardiol 2006;97: 52-60C

36. Statin safety: a perspective. Bandolier 2006;147. Available: www.medicine.ox.ac uk/bandolier/band147/b147-3.html (accessed 2008 Sep 6).

37. Mitka M. Contradictory findings ignite questions about blood glucose targets in diabetes. JAMA 2008;299:1413-5.

38. Action to Control Cardiovascular Risk in Diabetes Study Group. Effects of intensive glucose lowering in type 2 diabetes. N Engl J Med 2008;358:2545-59.

39. Farmer A, Wade A, Goyder E, et al. Impact of self monitoring of blood glucose in the management of patients with non-insulin treated diabetes: open parallel group randomised trial. BMJ 2007;335:132.

40. Heller SR. Self monitoring of blood glucose in type 2 diabetes. BMJ 2007;335: 105-6.

41. O'Kane MJ, Bunting B, Copeland M, et al. Efficacy of self monitoring of blood 
glucose in patients with newly diagnosed type 2 diabetes (ESMON study): randomised controlled trial. BMJ 2008;336:1174-7.

42. 2005/06 report card for the Ontario Drug Benefit Program. Toronto (ON): Ministry of Health and Long Term Care; 2008. Available: www.health.gov.on.ca/english /public/pub/ministry_reports/odb_report05/odb_rep_05_06.pdf (accessed 2008 Sep 6).

43. Hu G, Jousilahti P, Barengo NC, et al. Physical activity, cardiovascular risk factors, and mortality among Finnish adults with diabetes. Diabetes Care 2005;28:799-805.

44. Hu FB, Stampfer MJ, Solomon C, et al. Physical activity and risk for cardiovascular events in diabetic women. Ann Intern Med 2001;134:96-105.

45. Gregg EW, Gerzoff RB, Caspersen CJ, et al. Relationship of walking to mortality among US adults with diabetes. Arch Intern Med 2003;163:1440-7.

46. Church TS, LaMonte MJ, Barlow CE, et al. Cardiorespiratory fitness and body mass index as predictors of cardiovascular disease mortality among men with diabetes. Arch Intern Med 2005;165:2114-20.

47. Harris SB, Petrella RJ, Leadbetter W. Lifestyle interventions for type 2 diabetes. Relevance for clinical practice. Can Fam Physician 2003;49:1618-25.

48. Petrella RJ, Koval JJ, Cunningham DA, et al. Can primary care doctors prescribe exercise to improve fitness? The Step Test Exercise Prescription (STEP) project. Am J Prev Med 2003;24:316-22.

49. Kirk A, Mutrie N, MacIntyre P, et al. Effects of a 12-month physical activity counselling intervention on glycaemic control and on the status of cardiovascular risk factors in people with Type 2 diabetes. Diabetologia 2004;47:821-32.

50. Wolf AM, Conaway MR, Crowther JQ, et al. Translating lifestyle intervention to practice in obese patients with type 2 diabetes: Improving Control with Activity and Nutrition (ICAN) study. Diabetes Care 2004;27:1570-6.

51. Norris SL, Zhang X, Avenell A, et al. Long-term non-pharmacologic weight loss interventions for adults with type 2 diabetes. Cochrane Database Syst Rev 2005; (2):CD004095.

52. Wald NJ, Law MR. A strategy to reduce cardiovascular disease by more than $80 \%$. BMJ 2003;326:1419.

53. Feldman R. Simplified Therapeutic Intervention to Control Hypertension (STITCH) Trial. Final results. 2007 American Heart Association Meeting; 2007 Nov 8; Orlando (Flordia)

Correspondence to: Dr. Onil Bhattacharyya, Li Ka Shing

Knowledge Institute, St. Michael's Hospital, University of Toronto, 80 Bond St., Toronto ON M5B 1X2; fax 416 864-6057;

BhattacharyyaO@smh.toronto.on.ca

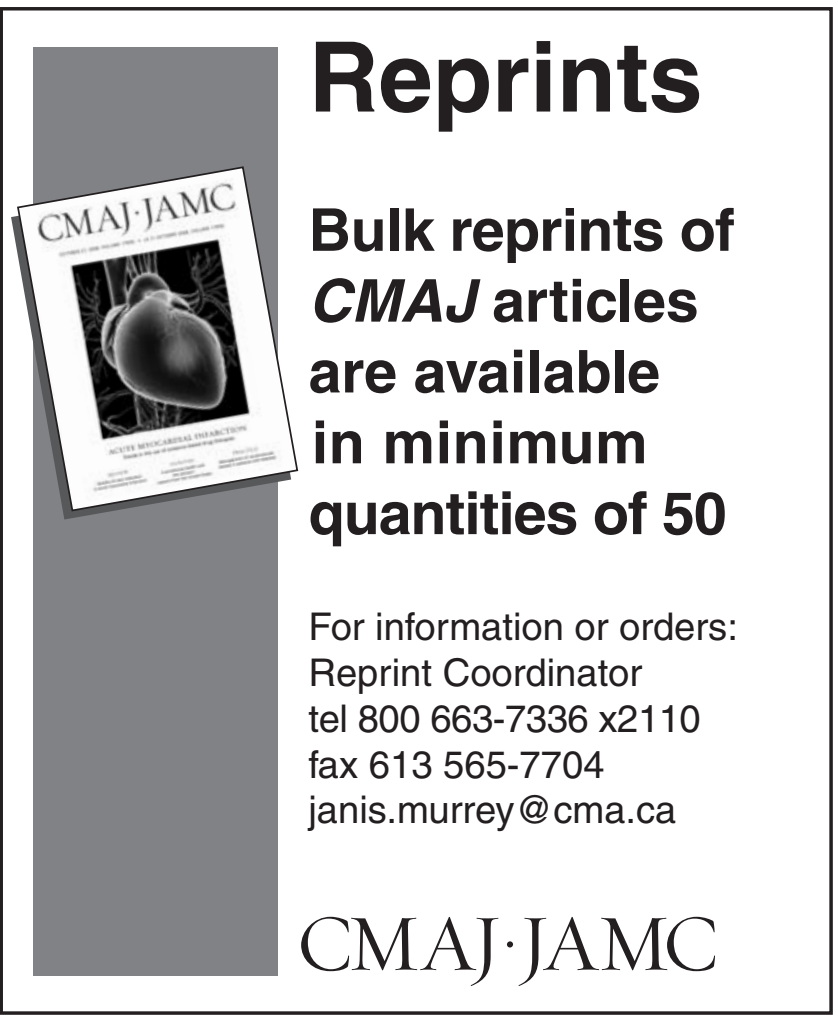

